To the Editors:

\title{
The Sri Lanka Clinical Trials Registry - five years young, and growing
}

Ceylon Medical Journal 2012; 57: 95-96

\section{Introduction}

The Sri Lanka Clinical Trials Registry (SLCTR) has completed five years of existence, and we take this opportunity to review its progress so far.

The global clinical trial regulatory landscape was changing at the dawn of the new millennium. There was widespread discontent over selective publication of positive trial results and concealment of negative results, leading to distortion of the body of evidence available for clinical decision making [1-3]. As a result, there was a growing call for more transparency in the conduct and public disclosure of clinical trials [1-3]. Mandatory registration of all clinical trials, before trial commencement and recruitment of participants, was seen as a possible mechanism to ensure that all trial data would be available in the public domain, thus enabling clinicians, patients, researchers and health care planners to make informed choices [1-3]. The call for trial registration, which started in the 1970s, gradually gathered momentum at the turn of the century $[4,5]$. A major turning point was the publication of two landmark articles in 2004 and 2005 by the International Committee of Medical Journal Editors (ICMJE), who demanded prospective registration of all trials if they were to be considered for publication $[3,6]$. The WHO strongly supported the call for mandatory trial registration, and established the International Clinical Trials Registry Platform (ICTRP) in 2005 to support and coordinate global efforts on trial registration [7]. The World Medical Association amended the Declaration of Helsinki at its 59th General Assembly in 2008 to state that every clinical trial must be registered in a publicly accessible database before recruitment of the first participant [8]. Prospective registration is now a mandatory requirement for the conduct of clinical trials.

The need for a national body for trial registration was first highlighted in a leading article in the Ceylon Medical Journal (CMJ) [9]. Recognising this need, the Sri Lanka Medical Association (SLMA) appointed a committee in August 2006 to establish and manage the SLCTR (the SLCTR Committee), with representation from the Ministry of Health, Postgraduate Institute of Medicine, universities, ethics review committees and other professional bodies. The SLCTR started functioning in November 2006, and was the first clinical trials registry in South Asia to commence operations. The first trial was registered with the SLCTR in February 2007. The establishment of the SLCTR has been described in detail previously $[10,11]$.
The SLCTR was recognized as a Primary Registry by the WHO-ICTRP in March 2008. It is a not-for-profit registry, with free access to clinicians, patients, researchers, health care planners, funding agencies and the public (http://www.slctr.lk). Data of all trials registered with the SLCTR are regularly uploaded to the ICTRP, and are thus available to the public worldwide via the ICTRP Clinical Trials Search Portal. Over the last five years, 68 clinical trials have been registered with the SLCTR, and four of them were international multi-centre trials. Of these, 39 trials have been completed, and two trials have stopped recruitment before completion. Fourteen trial applications were not accepted for registration, with delayed application after trial commencement being the main reason. During this period, 15 trials conducted in Sri Lanka have been registered with a trial registry in the WHO-ICTRP Registry Network other than the SLCTR.

From its inception, the SLCTR has striven to meet the stringent standards expected of an international clinical trials registry as specified by the WHO-ICTRP, and has continued to improve as the ICTRP requirements have evolved over the years. It updates trial records with progress reports and protocol changes, maintains and updates its standard operational procedures, and carries out internal audits of data quality and operational processes. The website has undergone several modifications, and is now being completely revamped.

The SLCTR is managed by the SLMA, and the SLCTR Committee advises on operational activities and policy matters. It works in close collaboration with the Ministry of Health, professional organisations, ethics review committees and other stakeholders in clinical research. The Ministry of Health has supported the SLCTR from its inception, and has provided the political commitment that is vital for the success of trial registration. Establishment of the SLCTR has, in turn, helped to improve research transparency at a national level, and augmented the Ministry's efforts to ensure the conduct of ethical and scientific research. The clinical trial landscape is changing in Sri Lanka, with a resurgent interest in conduct of clinical trials locally, and an influx of international collaborative research. This has brought into focus the need for improved ethical oversight of clinical research and better safeguards for participants. The Ministry of Health has taken several steps to strengthen the regulatory mechanisms governing clinical research, and the SLCTR has been an active participant in these initiatives. 
Awareness on clinical trial registration is a key ingredient for the success of the drive for prospective trial registration. The SLCTR has taken several steps to improve awareness on trial registration among the scientific community, with presentations at several scientific meetings across the country, publication of articles [10-12], and regular updates in newsletters of professional organizations. In addition to clinicians and researchers, patients and the public need to be aware of the concept of trial registration, and how information can be accessed on any clinical intervention. This is an area that the SLCTR has to address with more concerted effort.

Over the last five years, the SLCTR has been successful in establishing a national platform for trial registration in Sri Lanka. But there is much more that needs to be done, in addition to improving research transparency. There is a clear need to improve awareness on clinical research, to build research capacity, and to ensure that clinical trials conducted in Sri Lanka meet the highest ethical and scientific standards. The SLCTR is committed to working with its partner stakeholders to achieve these goals, and create a research environment that the country can be proud of.

\section{References}

1 Dickersin K, Rennie D. Registering clinical trials. Journal of the American Medical Association 2003; 290: 516-23.

2 Gotzsche PC. Multiple publication of reports of drug trials. European Journal of Clinical Pharmacology 1989; 36: 42932.

U K Ranawaka ${ }^{1}$, C Wanigatunga ${ }^{2}$, A de Abrew ${ }^{3}$, M Wimalachandra ${ }^{3}$, C Goonaratna $^{4}$

${ }^{1}$ Faculty of Medicine, University of Kelaniya, ${ }^{2}$ Faculty of Medical Sciences, University of Sri Jayewardenepura, ${ }^{3}$ Faculty of Medicine, University of Colombo, and ${ }^{4}$ Ceylon Medical College Council, Faculty of Medicine, University of Colombo

Correspondence: UKR, e-mail: <udayaran@yahoo.com>. Received 17 May 2012 and revised version accepted 22 May 2012. Competing interests: none declared.
3 De Angelis CD, Drazen JM, Frizelle FA, et al. Clinical trials registration. A statement from the International Committee of Medical Journal Editors. Journal of the American Medical Association 2004; 292: 1363-4.

4 Chalmers TC. Randomise the first patient! New England Journal of Medicine 1977; 296: 107.

5 Simes RJ. Publication bias: the case for an international registry of clinical trials. Journal of Clinical Oncology 1986; 4: 1529-41.

6 De Angelis CD, Drazen JM, Frizelle FA, et al. Is this clinical trial fully registered? A statement from the International Committee of Medical Journal Editors. New England Journal

7 International Clinical Trials Registry Platform (ICTRP). Geneva: World Health Organization. http://www.who.int/ ictrp/about/details/en/index.html. (Accessed 12 May 2012).

8 Declaration of Helsinki. Ethical principles for medical research involving human subjects. World Medical Association. http://www.wma.net/en/30publications/ 10policies/b3. (Accessed 12 May 2012).

9 Goonaratna C. Mandatory registration of clinical trials. Ceylon Medical Journal 2005; 50: 139-41.

10 Ranawaka UK, Goonaratna C. Sri Lanka Clinical Trials Registry. Ceylon Medical Journal 2007; 52: 117-9.

11 Ranawaka UK, Goonaratna C. Establishing the Sri Lanka Clinical Trials Registry. Journal of Evidence Based Medicine 2009; 2: 29-31.

12 Ranawaka UK, Goonaratna C. Sri Lanka Clinical Trials Medicine 2011; 4: 179-81. of Medicine 2005; 352: 2436-8. Registry - moving forward. Journal of Evidence Based 